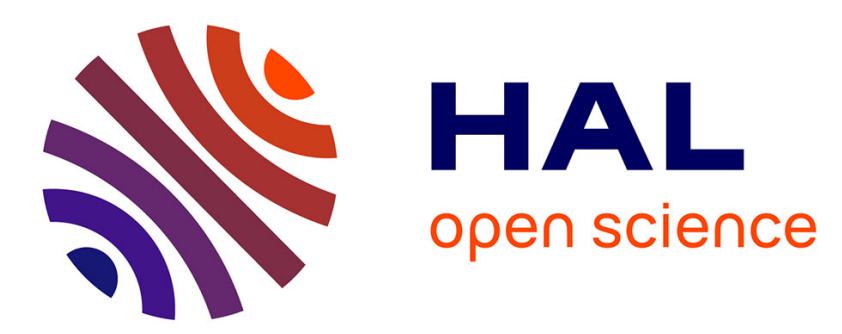

\title{
A thermodynamic description of the adsorption-induced structural transitions in flexible MIL-53 metal-organic framework
}

\author{
François-Xavier Coudert, Anne Boutin, Alain H. Fuchs
}

\section{To cite this version:}

François-Xavier Coudert, Anne Boutin, Alain H. Fuchs. A thermodynamic description of the adsorption-induced structural transitions in flexible MIL-53 metal-organic framework. Molecular Physics, 2014, 112 (9-10), pp.1257-1261. 10.1080/00268976.2014.889325 . hal-02116918

\author{
HAL Id: hal-02116918 \\ https://hal.science/hal-02116918
}

Submitted on 1 May 2019

HAL is a multi-disciplinary open access archive for the deposit and dissemination of scientific research documents, whether they are published or not. The documents may come from teaching and research institutions in France or abroad, or from public or private research centers.
L'archive ouverte pluridisciplinaire $\mathbf{H A L}$, est destinée au dépôt et à la diffusion de documents scientifiques de niveau recherche, publiés ou non, émanant des établissements d'enseignement et de recherche français ou étrangers, des laboratoires publics ou privés. 


\title{
A Thermodynamic Description of the Adsorption Induced Structural
} Transitions in Flexible MIL-53 Metal-Organic Framework

\author{
François-Xavier Coudert, ${ }^{*, a}$ Anne Boutin, ${ }^{\mathrm{b}}$ Alain H. Fuchs, ${ }^{*, \mathrm{a}}$ \\ ${ }^{a}$ Institut de Recherche de Chimie Paris, CNRS - Chimie ParisTech, 11 rue Pierre et \\ Marie Curie, 75005 Paris, France. \\ ${ }^{b}$ Département de Chimie, École Normale Supérieure, CNRS-ENS- UPMC, 24 rue \\ Lhomond, 75005 Paris, France.
}

Email address: fx.coudert@chimie-paristech.fr ; alain.fuchs@cnrs-dir.fr

We briefly review the concept of Temperature-Loading phase diagram that we derived from an osmotic statistical ensemble analysis of the adsorption induced structural transitions observed in the MIL-53 family of metal-organic framework nanoporous materials. We highlight the generic nature of this diagram and comment on the occurrence of breathing transitions depending on the guest fluid, the framework functionalization and the nature of the metal centre. 


\section{Introduction}

Among the wide class of Metal-Organic Frameworks (MOFs) and other related open framework hybrid materials, the subclass of Soft Porous Crystals (SPCs) has attracted a lot of attention in the past few years. According to Kitagawa, who introduced this notion, "soft porous crystal are defined as porous solids that possess both a highly ordered network and structural transformability. They are bistable or multistable crystalline materials with long range structural ordering, a reversible transformability between states and permanent porosity". It has been recently shown that the "softness" of these materials, which was somewhat ill-defined up to now, actually stems from a highly anisotropic mechanical (or elastic) behaviour, with some directions of the crystal exhibiting very low Young's modulus and shear modulus. ${ }^{2}$

SPCs exhibit large-amplitude structural deformations induced by various external stimuli such as temperature, mechanical pressure, or guest adsorption. Guest-induced structural transitions include the gate adsorption and the breathing phenomena. Gateadsorption typically involves an abrupt structural transition between a nonporous state and a porous crystalline host that is induced by gas adsorption. ${ }^{3,4}$ The breathing phenomenon consists of two successive adsorption-induced crystal-to-crystal transformations, from a large pore (lp) state to a narrow pore (np) state, and back again to the lp structure. ${ }^{5,6}$

In a series of articles, the present authors have investigated the equilibrium thermodynamics of adsorption-induced and pressure induced breathing transitions using an analytical approach based on the osmotic statistical ensemble. Some key questions were answered, such as the prediction of the occurrence or absence of guest-induced breathing for a given guest-host system, ${ }^{7,8,9}$ and the successful derivation of a \{temperature-gas pressure\} phase diagram for the several gases (xenon, methane, carbon dioxide)/MIL-53 systems ${ }^{10,11,12}$ as well as breathing phase diagram for binary mixtures of methane and carbon dioxide/MIL-53. ${ }^{13}$ The physical mechanism of the breathing transitions was addressed by viewing the adsorption-induced stress exerted on the material as the stimulus that triggers breathing transitions, in a way similar to lp-tonp structural transitions can be induced by mechanical compression. This new view of the breathing that we proposed implies that the structural transitions in SPCs takes place when the stress reaches a certain critical threshold. ${ }^{14,15}$ Though the exact chemical origins of this stress have not yet been fully uncovered, it is similar in nature to the 
elastic limit (or yield strength) in classical mechanics, where the deformation stops being quasistatic. For example, a recent molecular simulation study has shed some light on the pressure-induced amorphization of Zeolitic Imidazolate Framework ZIF-8: ${ }^{16}$ the amorphization transition in this material was shown to be the result of pressure-induced softening, until the pressure reached a value at which the crystal was not mechanically stable (Born's stability criteria no longer hold). Although it has not been equivocally demonstrated, we envision that a similar mechanism might be in play in the breathing of MIL-53.

This hypothesis of a "critical stress threshold" enabled to explain, at least qualitatively, the hysteresis phenomenon observed in the breathing transitions: when approaching the structural transition, the material is in two different phases on the adsorption and desorption branches, whose critical stress need not be equal (it was demonstrated, for example, that other mechanical properties such as the bulk modulus vary between the two phases ${ }^{15}$ ). Moreover, this concept was used as a basis for building a multiscale dynamic model in order to capture the dynamics of adsorption-induced deformation and structural transformation in MIL-53 based on the coupling of the host-guest adsorption interactions with the elastic response of the three-dimensional framework of a given geometry. ${ }^{17,18}$ Finally the issue of multistability and intermediate structures was recently addressed, using the osmotic potential approach. ${ }^{19}$

In this paper we briefly review the equilibrium Temperature-Loading phase diagrams (i.e. the \{temperature-gas pressure $\}$ diagrams) that were computed for different guestMIL-53 systems. We highlight the generic nature of this diagram and comment on the occurrence of breathing transitions depending on the guest fluid, the framework functionalization and the nature of the metal centre.

\section{Temperature-Loading diagrams for several guest-MIL-53(AI) systems}

We have used a model based on the so-called osmotic ensemble, ${ }^{7}$ which is the appropriate statistical ensemble to describe fluid adsorption in a flexible porous material. Since the MIL-53 material exhibits clear structural transitions between two metastable framework structures, namely the "narrow pore", np phase and the "large pore", Ip phase (see Figure 1), we were able to use a simplified osmotic sub-ensemble to describe the equilibrium between host structures upon fluid adsorption. ${ }^{7,8}$ Langmuir fits of the experimental isotherms served as approximations to the rigid host isotherms in 
both the lp and $\mathbf{n p}$ structures. We showed that, at a fixed temperature, the occurrence of breathing (or other structure transitions) upon adsorption could be rationalized by three key parameters with clear physical meaning: the affinity of the fluid for each phase (as described by the Langmuir's Henry constant); the capacity (or saturation uptake) of fluid in each phase; the free energy difference between the empty phases.
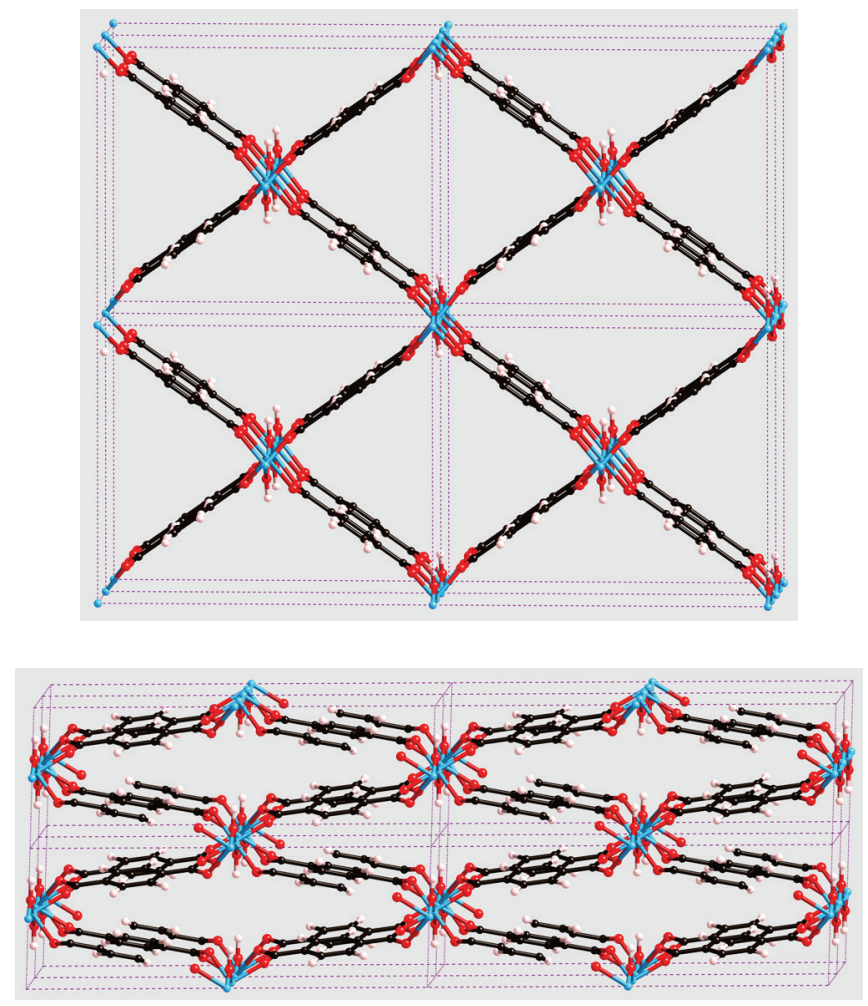

Figure 1. Representation of the metastable lp (top) and np (bottom) structures of the MIL-53(Al) material, as a $2 \times 2 \times 2$ supercell viewed along the axis of the unidimensional channels.

In the first study of this series, performed on the $\{$ Xenon- MIL-53 (Al) $\}$ system, ${ }^{10}$ we have used stepped isotherms at two temperatures to determine the transition enthalpy and entropy of the empty host material, as well as the free energy difference between the empty lp and the np structures. Not unexpectedly, the lp form was predicted to be the most stable one at room temperature, while the np structure becomes the most stable one below $203 \mathrm{~K}$. One of the interests of the osmotic thermodynamic model is that it enables to compute equilibrium thermodynamic data for the bare host material using thermodynamic adsorption data only. 
The osmotic model, with the use of the fits performed on the experimental adsorption isotherms, enables to investigate the full temperature-loading phase diagram of $\{\mathrm{Xe}$, MIL-53(Al)\}. By solving the osmotic thermodynamic equations numerically, we determined for each temperature, whether breathing takes place and, if so, what the transition pressures are. The predicted Temperature-Xenon gas Pressure diagram is shown in Figure 2.

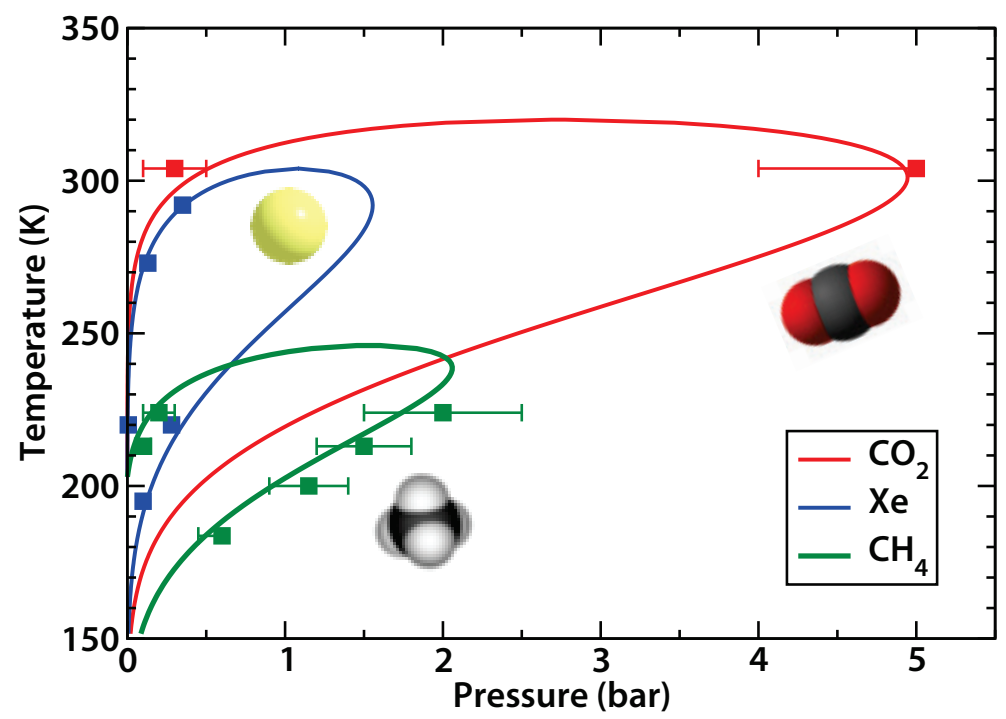

Figure 2. Comparison of the Temperature-gas Pressure phase diagrams for $\mathrm{CO}_{2}$ (red), $\mathrm{CH}_{4}$ (green) and Xe (blue) adsorption in MIL-53 (Al). ${ }^{12}$ Experimental transition pressures are indicated by square symbols.

The Ip phase was found to be stable at high temperature and again at lower temperature. There is an intermediate np phase stability domain for xenon pressure lower than a limiting pressure of around 1.6 bar. This result is reminiscent of the re-entrant behavior observed in some liquid crystals. ${ }^{20}$ As noted above, however, the low-temperature stable phase at zero pressure (empty material) is the np phase.

The phase diagrams for the $\left\{\mathrm{CO}_{2}, \mathrm{MIL}-53(\mathrm{Al})\right\}$ and $\left\{\mathrm{CH}_{4}, \mathrm{MIL}-53(\mathrm{Al})\right\}$ are also shown in Fig. 2. ${ }^{12}$ All three diagrams have in common the equilibrium np-lp temperature $\mathrm{T}_{0}$ of $203 \mathrm{~K}$ at zero pressure. The initial slope of the transition curve is proportional to the logarithm of $\left(K_{\mathrm{np}} / K_{\mathrm{lp}}\right)$, the ratio of adsorption affinities in the two structures, and is thus strictly positive since the affinity of the guest adsorbate for the closed form of the framework is higher than for the open form. ${ }^{7,10}$ The condition $K_{\mathrm{np}} / K_{\mathrm{lp}}>1$ thus favors the 
closed np phase, and consequently the phase transition temperature increases with the gas loading (i.e. the stability domain of the $\mathbf{n p}$ phase increases with $P_{\text {adsorbate) }}$ ). At higher temperature, the transition free energy increases, and it becomes more and more difficult to maintain the np form as the most stable one. This causes the observed bending of the transition line. For obvious entropy reasons, the lp phase will eventually become more stable at high temperature, regardless of the gas loading. This situation is also true at high pressure. As the adsorbate pressure increases, at any temperature, the lp structure will eventually become more stable than the np one because it can accommodate a higher loading of guest molecules. Since the lp phase is the most stable one at high enough temperature as well as at high adsorbate pressure, one has to conclude that the stability domain of the np phase should be limited in adsorbate pressure $\left(P_{\max } \sim 5\right.$ bar in the case of carbon dioxide, 2 bar in the case of methane and 1.6 bar in the case of xenon, see Figure 2).

The above thermodynamic considerations are very general and obviously not limited to the special case of one or the other gas adsorption. The condition $K_{\mathrm{np}} / K_{\mathrm{lp}}>1$ is expected to hold true for all the simple guest molecules that have been investigated so far. This means that there should be a range of temperatures above the equilibrium npIp transition temperature of the bare MIL-53(Al) material (203 K in our model, subject to an estimated uncertainty of $\pm 10 \mathrm{~K}$ ) where the initially empty open structure contracts upon guest molecule adsorption. The fact that this has not been observed in some cases at room temperature simply means that the transition line maximum in this system is below the room temperature. The present findings for methane clearly confirm these predictions.

The difference in the stability domain of the np phase in the three different cases can be qualitatively understood as follows. For each system, there is a temperature $T_{\max }$ (see Figure 3a), above which gas adsorption does not induce the lp->np phase transition anymore. Since the driving force for the closure of the lp structure is the guest-host interaction, which induces the cell contraction, one may simply write: $k T_{\max } \sim \Delta H_{\mathrm{ads}, \mathrm{p} \text {. }}$ The increase in $\mathrm{T}_{\max }$ in going from $\mathrm{CH}_{4}$ to $\mathrm{Xe}$ and $\mathrm{CO}_{2}$ can then simply be explained by the increase in adsorption enthalpy in the Ip phase. While $\mathrm{P}_{\max }$ is approximately the same for $\mathrm{CH}_{4}$ and $\mathrm{Xe}$, its value for $\mathrm{CO}_{2}$ is larger by a factor of $\sim 3$. This difference can be accounted for by a larger interaction energy between guest molecules in the case of carbon dioxide, due their quadrupole moments. This causes a stronger ordering of the 
$\mathrm{CO}_{2}$ molecules in the np phase and increases the stability domain of this phase.

In figure $3 b$ we sketch the three phase diagrams in reduced units of $\left(T / T_{\max }\right)$ and $\left(\mathrm{P} / \mathrm{P}_{\max }\right)$. Some sort of a "universal" phase diagram seem to come out of this presentation. This confirms the existence of a generic temperature-loading phase diagram, as long as the adsorbate is a simple gas such as methane, carbon dioxide or xenon.
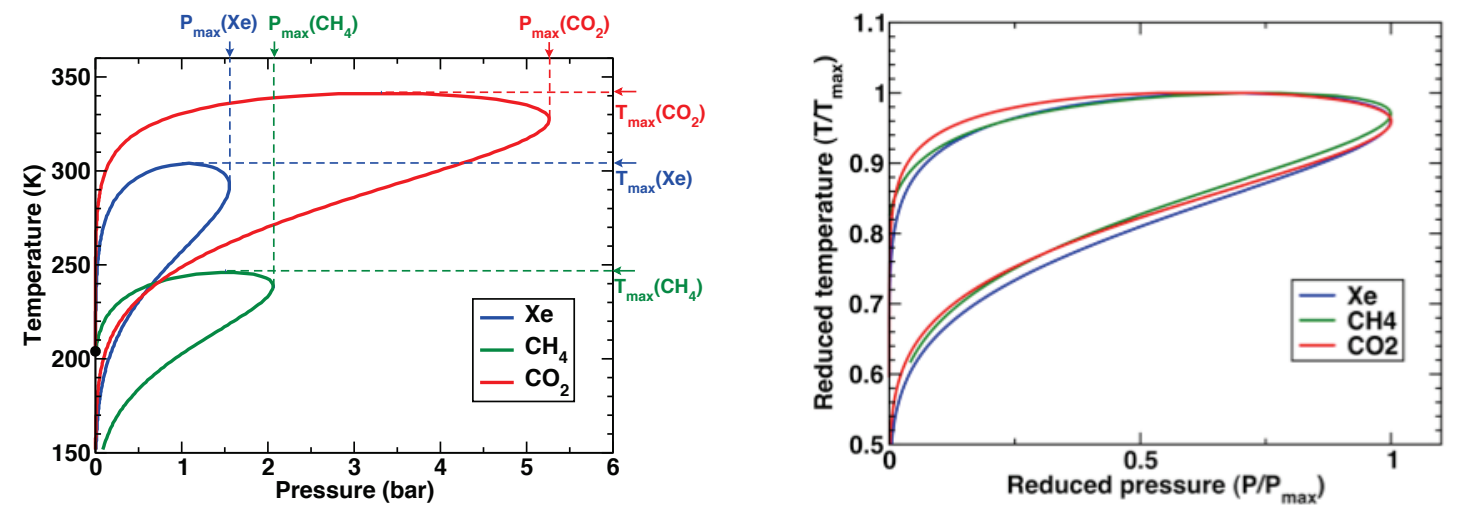

Figure 3. Left: Comparison of the temperature-vapour pressure phase diagrams for $\mathrm{CO}_{2}$ (red), $\mathrm{CH}_{4}$ (green), and $\mathrm{Xe}$ (blue) adsorption in MIL-53(Al), with the extremal temperatures and pressures marked. Right: same three phase diagrams in reduced units of $\left(T / T_{\max }\right)$ and $\left(P / P_{\max }\right)$. Left figure is reproduced with permission from ref. 12, (C) 2010 American Chemical Society.

It must be recalled at this stage that the osmotic model predicts the conditions of thermodynamic stability at full equilibrium and does not take into account hysteresis effects. Hysteresis was systematically encountered in all reported MIL-53 experiments and often leads to some complicated mixtures of phases. ${ }^{21}$ This model describes what would happen in a homogeneous system at full equilibrium. The osmotic model is aimed at describing the thermodynamics behind the scene. The hysteresis issue is being addressed in some of our other studies. ${ }^{14-19}$

Finally one should mention that the osmotic model was extended to predict the behaviour of fluid mixtures adsorbing in flexible nanoporous materials by proposing an analytical scheme called OFAST (Osmotic Framework Adsorbed Solution Theory). ${ }^{13}$ Figure 4 represents the $\left\{\mathrm{CO}_{2} / \mathrm{CH}_{4}, \mathrm{MIL}-53(\mathrm{Al})\right\}$ diagrams for different values of the carbon dioxide mole fraction. We can see that, in addition to the re-entrant nature of the 
phase diagrams themselves in $(T, P)$ space, there is also a nonmonotonic evolution of the maximal temperature and pressure of the np phase as a function of the mixture's composition. This unexpected result, first hypothesized on a purely theoretical thermodynamic basis, ${ }^{9}$ was later proven true by experimental measurements. ${ }^{13}$

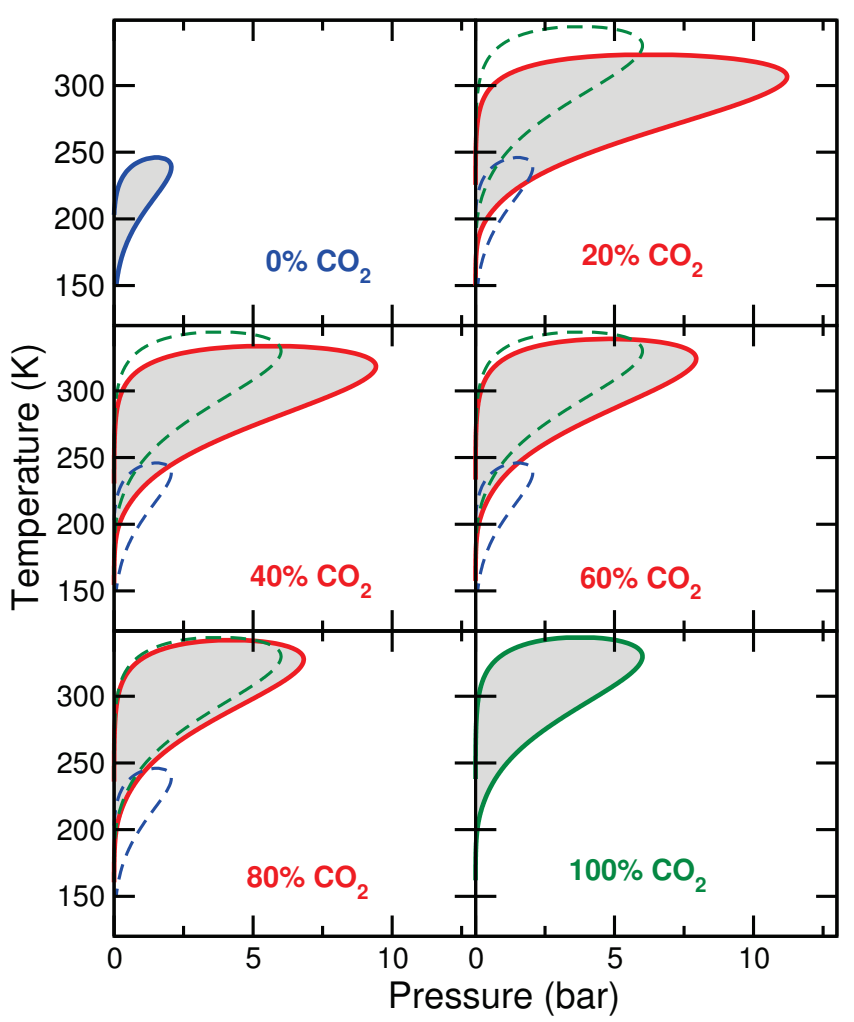

Figure 4. Temperature-pressure phase diagram of MIL-53(Al) upon adsorption of a $\mathrm{CO}_{2} / \mathrm{CH}_{4}$ mixture, with increasing $\mathrm{CO}_{2}$ molar fraction. Dashed lines correspond to pure component diagrams: $\mathrm{CO}_{2}$ in green, $\mathrm{CH}_{4}$ in blue. Reproduced with permission from ref. 13, (C) 2011 American Chemical Society.

\section{Temperature-Loading phase diagrams for some other guest-MIL-53 materials systems}

The osmotic Temperature-Loading phase diagram can be used to study the effect of changes in the material's composition on the breathing behaviour. We have performed an experimental and theoretical study of carbon dioxide adsorption in aminofunctionalized MIL-53(Al), jointly with the group of Denayer. ${ }^{11}$ In Figure 5 the $\left\{\mathrm{CO}_{2}\right.$, amino-MIL-53(Al)\} diagram is compared with the «regular» MIL-53(Al) diagram. The equilibrium np-lp temperature $\mathrm{T}_{0}$ is slightly different (203 $\mathrm{K}$ for regular MIL-53 and $226 \mathrm{~K}$ for amino-MIL-53), and the initial slope of the transition line is higher in the 
case of the amino-MIL system. Even though the overall shape of the temperature-gas pressure phase diagram is similar, there is a spectacular difference in the stability domain of the np structure, which can be accounted for by the increased stability of this form due to the presence of the amino groups in the structure. The strong affinity of $\mathrm{CO} 2$ molecules in the $\mathbf{n p}$ form leads to a larger value of $\mathrm{K}_{\mathrm{np}} / \mathrm{K} 1 \mathrm{p}$ for the amino-MIL material compared to the regular MIL-53(Al) and enlarges the stability domain of the np form.

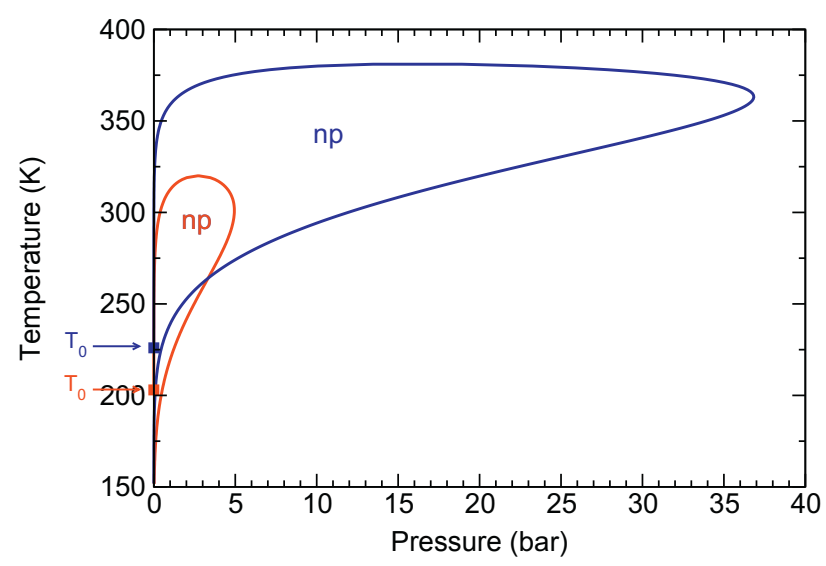

Figure 5. Temperature- $\mathrm{CO}_{2}$ pressure phase diagrams for regular MIL-53(Al) (red) and amino-MIL-53(Al) (blue) materials. The $T_{0}$ are the equilibrium phase transition temperature between the $\mathbf{l p}$ and $\mathbf{n p}$ phases at zero pressure (i.e. the empty material) for both materials. Modified with permission from ref. 11, (C) 2010 Elsevier Inc.

An even more drastic change in the MIL-53 composition consists in replacing the aluminium center by a gallium atom. MIL-53 (Ga) was synthesized and characterized by the group of Férey, ${ }^{22}$ and then further investigated by Boutin and coworkers. ${ }^{23}$ Breathing transitions were found to take place upon several gas adsorption ${ }^{22}$ but the stable phase at room temperature was found to be the np narrow pore structure. ${ }^{22,23} \mathrm{~A}$ recent quantum chemistry investigation by Coudert and coworkers allowed to understand that $\mathbf{n p}$ phase was more stable in the case of MIL-53 (Ga), because of the more diffuse orbitals of Ga compared to $\mathrm{Al}^{24}{ }^{24}$ Thus the $\mathbf{n p}$ phase is the stable structure at room temperature (rather than lp as Al-MIL53). The effect of replacing Al by Ga is simply to increase the value of $\mathrm{T}_{0}$, the $\mathbf{n p}$-lp equilibrium phase transition temperature of the empty MIL-53 framework. The whole Temperature-Loading phase diagram is thus 
switched to higher temperature.

\section{Conclusion}

The temperature-loading phase diagram of the flexible MIL-53 family of materials is a useful tool to understand the behaviour of adsorption induced breathing structural transitions. Whether or not these transitions take place upon adsorption of a particular gas or gas mixture depend on the relative location of the stability regions of the $\mathbf{n p}$ and Ip states in the system's $(T, P, x)$ space, where $T$ is the temperature, $P$ is the adsorbate gas pressure and $x$ is a mole fraction describing the gas composition. Even though this is just an equilibrium thermodynamic view of the breathing process, which means for instance that it lacks the hysteresis effects that take place in the experiments, this temperature-loading concept has allowed to demonstrate that the breathing effect in MIL-53 is a very general phenomenon, that should be observed in some region of the $(T, P)$ space whatever the guest molecule, since it is expected that the affinity of an adsorbate for the closed $\mathbf{n p}$ form of the framework will always be higher than for the open Ip structure. We also believe that this very general model can provide a useful tool for experimentalists to tune their experimental conditions. As such, the phase diagrams described here and the thermodynamic models developed to build them naturally complement the other approaches used in the field of flexible porous materials, namely poromechanics $^{25,26}$ (at the macroscopic scale) and atomistic molecular simulation (at the microscopic scale) $;^{27,28,29,30}$ the advantages and downsides of all three methods were recently reviewed in ref. 31 .

\section{Acknowledgements}

This short review paper is dedicated to Professor Pierre Turq, on the occasion of his 70th birthday. Pierre was a pioneer in introducing statistical mechanics and molecular simulations at all scales in France, in the field of chemistry and physical chemistry, and the French community owes him a lot.

The authors acknowledge funding from the Agence Nationale de la Recherche under the project “'SOFT-CRYSTAB”' (ANR-2010-BLAN-0822).

\section{References}


${ }^{1}$ S. Horike, S. Shimomura and S. Kitagawa, Nature Chem. 1, 695 (2009)

${ }^{2}$ A. U. Ortiz, A. Boutin, A. H. Fuchs and F.-X. Coudert, Phys. Rev. Lett. 109, 195502 (2012)

${ }^{3}$ D. Li and K. Kaneko, Chem. Phys. Lett. 335, 50 (2001)

${ }^{4}$ R. Kitaura, K. Seki, G. Akiyama and S. Kitagawa, Angew. Chem. Int. Ed. 42, 428 (2003).

${ }^{5}$ G. Férey and C. Serre, Chem. Soc. Rev. 38, 1380 (2009).

${ }^{6}$ C. Serre, F. Millange, C. Thouvenot, M. Nogues, G. Marsolier, D. Louer and G. Férey, J. Am. Chem. Soc. 124, 13519 (2002).

${ }^{7}$ F.-X. Coudert, M. Jeffroy, A. H. Fuchs, A. Boutin and C. Mellot-Draznieks, J. Am. Chem. Soc. 130, 14294 (2008)

${ }^{8}$ F.-X. Coudert, C. Mellot-Draznieks, A. H. Fuchs and A. Boutin, J. Am. Chem. Soc. 131, 3442 (2009).

${ }^{9}$ F.-X. Coudert, Phys. Chem. Chem. Phys. 12, 10904 (2010).

${ }^{10}$ A. Boutin, M.-A. Springuel-Huet, A. Nossov, A. Gedeon, T. Loiseau, C. Volkringer, G. Férey, F.-X. Coudert and A. H. Fuchs, Angew. Chem. Int. Ed. 48, 8314 (2009).

${ }^{11}$ A. Boutin, S. Couck, F.-X. Coudert, P. Serra-Crespo, J. Gascon, F. Kapteijn, A. H. Fuchs and J. F. M. Denayer, Microporous Mesoporous Mater. 140, 108 (2011).

${ }^{12}$ A. Boutin, F.-X. Coudert, M. A. Springuel-Huet, A. V. Neimark, G. Férey and A. H. Fuchs, J. Phys. Chem. C 114, 22237 (2010).

${ }^{13}$ A. U. Ortiz, M. A. Springuel-Huet, F.-X. Coudert, A. H. Fuchs and A. Boutin, Langmuir 28, 494 (2012).

${ }^{14}$ A. V. Neimark, F.-X. Coudert, A. Boutin and A. H. Fuchs, J. Phys. Chem. Lett. 1, $445(2010)$

${ }^{15}$ A. V. Neimark, F.-X. Coudert, C. Triguero, A. Boutin, A. H. Fuchs, I. Beurroies and R. Denoyel, Langmuir 27, 4734 (2011)

${ }^{16}$ A. U. Ortiz, A. Boutin, A. H. Fuchs and F.-X. Coudert, J. Phys. Chem. Lett., 2013, 4, 1861-1865.

${ }^{17}$ C. Triguero, F.-X. Coudert, A. Boutin, A. H. Fuchs and A. V. Neimark, J. Phys. Chem. Lett. 2, 2033 (2011)

${ }^{18}$ C. Triguero, F.-X. Coudert, A. Boutin, A. H. Fuchs and A. V. Neimark, J. Chem. Phys. 137, 18702 (2012)

${ }^{19}$ D. Bousquet, F.-X. Coudert, A.G.J. Fossati, A.V. Neimark, A.H. Fuchs and A. Boutin, J. Chem. Phys. 138, 174706 (2013)

${ }^{20}$ P. G. de Gennes and J. Prost, The Physics of Liquid Crystals, Oxford University Press, (2001)

${ }^{21}$ F. Salles, A. Ghoufi, G. Maurin, R.G. Bell, C. Mellot-Draznieks, P.L. Llewellyn, C. Serre and G. Férey, Angew. Chem., Int. Ed. 47, 8487 (2008)

${ }^{22}$ C. Volkringer, T. Loiseau, N. Guillou, G. Férey, E. Elkaïm and A. Vimont, Dalton Trans. 2241, (2009) 
${ }^{23}$ A. Boutin, D. Bousquet, A.U. Ortiz, F.-X. Coudert, A.H. Fuchs, A. Balandras, G. Weber, I. Bezverkhyy, J.-P. Bellat, G. Ortiz, G. Chaplais, J.-L Paillaud, C. Marichal, H. Nouali and J. Patarin, J. Phys. Chem. C 117, 8180 (2013)

${ }^{24}$ F.-X. Coudert, A.U. Ortiz, V. Haigis, D. Bousquet, A.H. Fuchs, A. Ballandras, G. Weber, I. Bezverkhyy, N. Geoffroy, J.-P. Bellat, G. Ortiz, G. Chaplais, J. Patarin and A. Boutin, J. Phys. Chem. C, submitted for publication.

${ }^{25}$ Brochard, L.; Vandamme, M.; Pellenq, R. J.-M. Poromechanics of Microporous Media. J. Mech. Phys. Solids 2012, 60, 606-622

${ }^{26}$ Mechanics and Physics of Porous Solids; Coussy, O., Ed.; John Wiley \& Sons: New York, 2010

${ }^{27}$ Ghoufi, A.; Maurin, G. J. Phys. Chem. C 2010, 114, 6496.

${ }^{28}$ Chen, L.; Mowat, J. P. S.; Fairen-Jimenez, D.; Morrison, C. A.; Thompson, S. P.; Wright, P. A.; Düren, T. J. Am. Chem. Soc., 2013, 135, 15763-15773

${ }^{29}$ Yot, P. G.; Ma, Q.; Haines, J.; Yang, Q.; Ghoufi, A.; Devic, T.; Serre,C.;Dmitriev,V.;Feŕey,G.;Zhong,C.;Maurin, G. Chem. Sci 2012, 3, 1100- 1104.

${ }^{30}$ Zang, J.; Nair, S.; Sholl, D. S. J. Chem. Phys. 2011, 134, 184103.

${ }^{31}$ F.-X. Coudert, A. Boutin, A. H. Fuchs and A. V. Neimark, J. Phys. Chem. Lett., 2013, 4, 3198-3205. 